\title{
Níveis de Lisina para Frangos de Corte nos Períodos de 22 a 42 e de 43 a 49 Dias de Idade, Mantendo a Relação Metionina + Cistina ${ }^{1}$
}

\section{Valene da Silva Amarante Júnior ${ }^{2}$, Fernando Guilherme Perazzo Costa ${ }^{3}$, Leilane da Rocha Barros $^{4}$, Germano Augusto Jerônimo do Nascimento ${ }^{4}$, Patrícia Araújo Brandão², José Humberto Vilar da Silva ${ }^{5}$, Walter Esfrain Pereira ${ }^{6}$, Ricardo Vianna Nunes ${ }^{7}$, Janaine Sena da Costa ${ }^{8}$, Marcelo Luís Gomes Ribeiro ${ }^{4}$}

RESUMO - Foram conduzidos dois experimentos com o objetivo de determinar as exigências de lisina para frangos de corte machos, da linhagem Ross, de 22 a 42 e de43 a 49 dias de idade, mantendo-se relação em 71\% de Met+cis:lisina. Utilizaram-se, em cada experimento, 900 frangos com peso médio inicial de 780 g aos 22 dias e de 2.556 g aos 43 dias de idade, distribuídos em um delineamento inteiramente casualizado, com seis tratamentos, seis repetições e 25 aves por unidade experimental. Os tratamentos consistiram de seis diferentes níveis de lisina, de acordo com a fase, mantendo-se a relação met + cis:lisina. As exigências nutricionais mínimas, exceto para lisina e para metionina + cistina, foram atendidas. Foram avaliados consumo de ração, ganho de peso, conversão alimentar, rendimento de carcaça e dos principais cortes, sendo as exigências de lisina estimadas por meio de modelos de regressão. Considerando-se os dados obtidos, as exigências nutricionais de lisina total foram estimadas em 1,140\% para máximo desempenho, no período de 22 a 42 dias de idade, e de 1,024\% para máximo desempenho e rendimento de carcaça e peito, no período de 43 a 49 dias de idade.

Palavras-chave: cortes nobres, desempenho, exigências nutricionais, frangos de corte, rendimento de carcaça

\section{Levels of Lysine for Broilers in the Periods from 22 to 42 and from 43 to 49 Days Old, Maintaining the Methionine + Cystine Ratio}

\begin{abstract}
Two experiments were conducted with the objective of evaluating the lysine requirements for Ross broilers, males, from 22 to 42 and 43 to 49 days old, maintaining $71 \%$ met + cys:lys ratio. Nine hundred broilers averaging initial body weight of $780 \mathrm{~g}$ at 22 days old and $2.556 \mathrm{~g}$ at 43 days old were used in each experiment and allotted to a completely randomized experimental design, with six treatments, six replicates and 25 birds by experimental unit. The treatments consisted of six different synthetic lysine levels, according to the phase and the fixed ratio. Minimum nutrition requirements, except lysine and met + cys, were met. Feed intake, body weight gain, feed gain ratio, carcass yield and the prime cuts yield were evaluated. The lysine requirements were estimated by the regression models. Considering the performance data, the nutritional requirements of total lysine were of $1.140 \%$ for maximum performance, in the period from 22 to 42 days old, and of $1.024 \%$ for maximum performance and carcass and breast yield, in the period from 43 to 49 days old.
\end{abstract}

Key Words: broilers, carcass yield, noble cuts, nutritional requirements, performance

\section{Introdução}

Atualmente, na avicultura industrial, o fator econômico tem sido o principal aspecto considerado para escolha dos ingredientes da ração. A fonte de proteína é um dos ingredientes mais caros no custo da ração, devendo, portanto, ser maximizado seu aproveitamento com produtos de qualidade. O conceito de proteína ideal tem sido aplicado como um modelo para maximizar o aproveitamento protéico, por meio do balanço ade- quado entre todos os aminoácidos essenciais. Entretanto, torna-se necessária a determinação das exigências nutricionais das aves, pois o excesso de aminoácidos implica em degradação da proteína, sendo o nitrogênio eliminado pela urina (Scott et al., 1982). Isso provoca o aumento do potencial poluente e prejuízo econômico, pois o nutriente não estaria sendo aproveitado eficientemente.

A lisina, apesar de não ser o primeiro aminoácido limitante para aves, é utilizada como aminoácido base

\footnotetext{
1 Parte da tese de Doutorado do primeiro autor.

2 Aluno do Programa de Doutorado Integrado em Zootecnia (UFRPE/UFC/UFPB).

3 Professor do Departamento de Zootecnia do CCA/UFPB. E.mail: fperazzo@cca.ufpb.br

4 Aluno do Programa de Pós-Graduação em Zootecnia - CCA/UFPB.

5 Professor do Centro de Formação e Tecnólogo - CFT/UFPB.

6 Professor do Departamento de Ciências Fundamentais e Sociais do CCA/UFPB.

7 Professor do Departamento de Zootecnia/UNIOESTE, M.C. Rondon, PR.

8 Aluno do curso de Zootecnia do CCA/UFPB.
} 
para cálculo da proteína ideal, visto que é utilizada quase que exclusivamente para formação de proteína corporal e não participa de interações metabólicas com outros aminoácidos. Além disso, em uma perspectiva analítica é o aminoácido mais simples de se analisar (Lemme, 2003). Entretanto, os experimentos que avaliam os níveis de lisina não consideram a relação com metionina+cistina, que também é de fundamental importância no desenvolvimento corporal dos frangos.

Sklan \& Noy (2004), estudando diferentes relações de aminoácidos na dieta verificaram que ocorre aumento na deposição de lisina, treonina e arginina, enquanto a deposição dos outros aminoácidos permanece constante, observando que a eficiência de deposição diminui com a idade.

Existe uma tendência atual de planejar experimentos relacionando o nutriente pesquisado com outros nutrientes da ração. Nascimento (2004) relata que alguns estudos demonstram respostas positivas quando o nível de outros aminoácidos mantém a mesma proporção com a variação dos níveis de lisina.

Portanto, o objetivo neste trabalho foi avaliar o desempenho de frangos de corte de 22 a 42 e de 43 a 49 dias de idade, submetidos a dietas contendo diferentes níveis de lisina, mantendo a mesma relação metionina+cistina.

\section{Material e Métodos}

Os experimentos foram conduzidos no Aviário Experimental do Departamento de Zootecnia do Centro de Ciências Agrárias da Universidade Federal da Paraíba. Os frangos foram alojados em um galpão de alvenaria coberto com bagaço de cana, com 36 boxes de dimensões de 1,40 x 1,80 m, divididos por mureta de alvenaria de $0,30 \mathrm{~cm}$ e piso de cimento.

Durante o período experimental, foi adotado o programa de luz contínua (24 horas de luz natural + artificial). As leituras de temperatura e de umidade máximas e mínimas foram registradas diariamente utilizando um termômetro de bulbos seco e úmido (Tabela 1).

Durante os períodos pré-inicial (1 a 7 dias) e inicial (8 a 21 dias), as aves receberam dietas comerciais com 23 e $21 \%$ de $P B$ e 2.930 e $3.050 \mathrm{kcal} / \mathrm{kg}$ de EM, respectivamente. As aves utilizadas nas duas fases experimentais (22 a 42 e 43 a 49 dias de idade) não foram as mesmas, sendo portanto, realizados dois experimentos distintos. Água e ração foram fornecidas à vontade.
Tabela 1 - Temperatura e umidade relativa, máximas e mínimas para as fases de 22 a 42 e 43 a 49 dias de idade

Table 1 - Maximum and minimum temperature and relative umidity from 22 to 42 and 43 to 49 days old

\begin{tabular}{|c|c|c|c|c|}
\hline \multirow{3}{*}{$\begin{array}{l}\text { Fase } \\
\text { (dias de idade) } \\
\text { Phase } \\
\text { (days old) }\end{array}$} & \multicolumn{2}{|c|}{$\begin{array}{l}\text { Temperatura } \\
\text { relativa }(\%)\end{array}$} & \multirow{2}{*}{\multicolumn{2}{|c|}{$\begin{array}{l}\text { Umidade } \\
\text { Humidity }\end{array}$}} \\
\hline & \multicolumn{2}{|c|}{ Relative temperature } & & \\
\hline & $\begin{array}{l}\text { Mínima } \\
\text { Minimum }\end{array}$ & $\begin{array}{c}\text { Máxima } \\
\text { Maximum }\end{array}$ & $\begin{array}{l}\text { Mínima } \\
\text { Minimum }\end{array}$ & $\begin{array}{c}\text { Máxima } \\
\text { Maximum }\end{array}$ \\
\hline 22 a 42 dias & 17,46 & 23,17 & 74 & 98 \\
\hline 43 a 49 dias & 17,41 & 22,50 & 72 & 98 \\
\hline
\end{tabular}

Para cada fase avaliada (22 a 42 e 43 a 49 dias), foram utilizados 900 frangos de corte machos da linhagem Ross, em um delineamento experimental inteiramente casualizado, com seis tratamentos (seis níveis de lisina mantendo-se relação de 71\% com Met + cis), seis repetições e 25 aves por unidade experimental. Os pesos iniciais das aves foram de $780 \mathrm{~g}$, para a fase de 22 a 42, e de 2.556 g, para a fase de 43 a 49 dias de idade. Os animais foram submetidos a dietas basais (Tabela 2), calculadas para atender as exigências preconizadas por Rostagno et al. (2000), exceto para lisina e para met + cis.

As dietas foram formuladas à base de milho e de farelo de soja e suplementadas com seis níveis de L-lisina: $\mathrm{HCl} 78 \%(0,00 ; 0,08 ; 0,16 ; 0,24 ; 0,32$ e $0,40 \%$ ), correspondendo aos níveis de 0,$936 ; 1,016$; 1,096; 1,176; 1,256 e 1,336\% de lisina total, no período de 22 a 42 dias e com 0,$00 ; 0,102 ; 0,204 ; 0,306 ; 0,408$ e $0,510 \%$, correspondendo aos níveis de 0,$850 ; 0,930$; 1,010; 1,090; 1,170 e 1,250\% de lisina total, no período de 43 a 49 dias. Os aminoácidos sintéticos foram introduzidos em substituição ao amido de milho da dieta. As exigências nutricionais mínimas, exceto para lisina, foram estimadas segundo as recomendações de Rostagno et al. (2000), mantendo-se, entretanto, a relação met + cis:lis em $71 \%-(0,664 / 0,936$; 0,721/1,016; 0,778/1,096; 0,835/1,176; 0,892/1,256; $0,949 / 1,336)$ e (0,603/0,850; 0,660/0,930; 0,717/1,010; $0,774 / 1,090,0,831 / 1,170$ e $0,888 / 1,250$ ) para as aves de 22 a 42 e 43 a 49 dias de idade, respectivamente.

Ao final de cada fase, foram avaliados consumo de ração, ganho de peso e conversão alimentar. Em seguida, após jejum de oito horas, foram abatidas quatro aves por unidade experimental, com peso médio da parcela, para determinação dos rendimentos de carcaça, em relação ao peso vivo das aves ao 
Tabela 2 - Composição percentual e calculada da dieta basal ${ }^{1}$

Table 2 - Calculated (\%) composition of the basal diet ${ }^{1}$

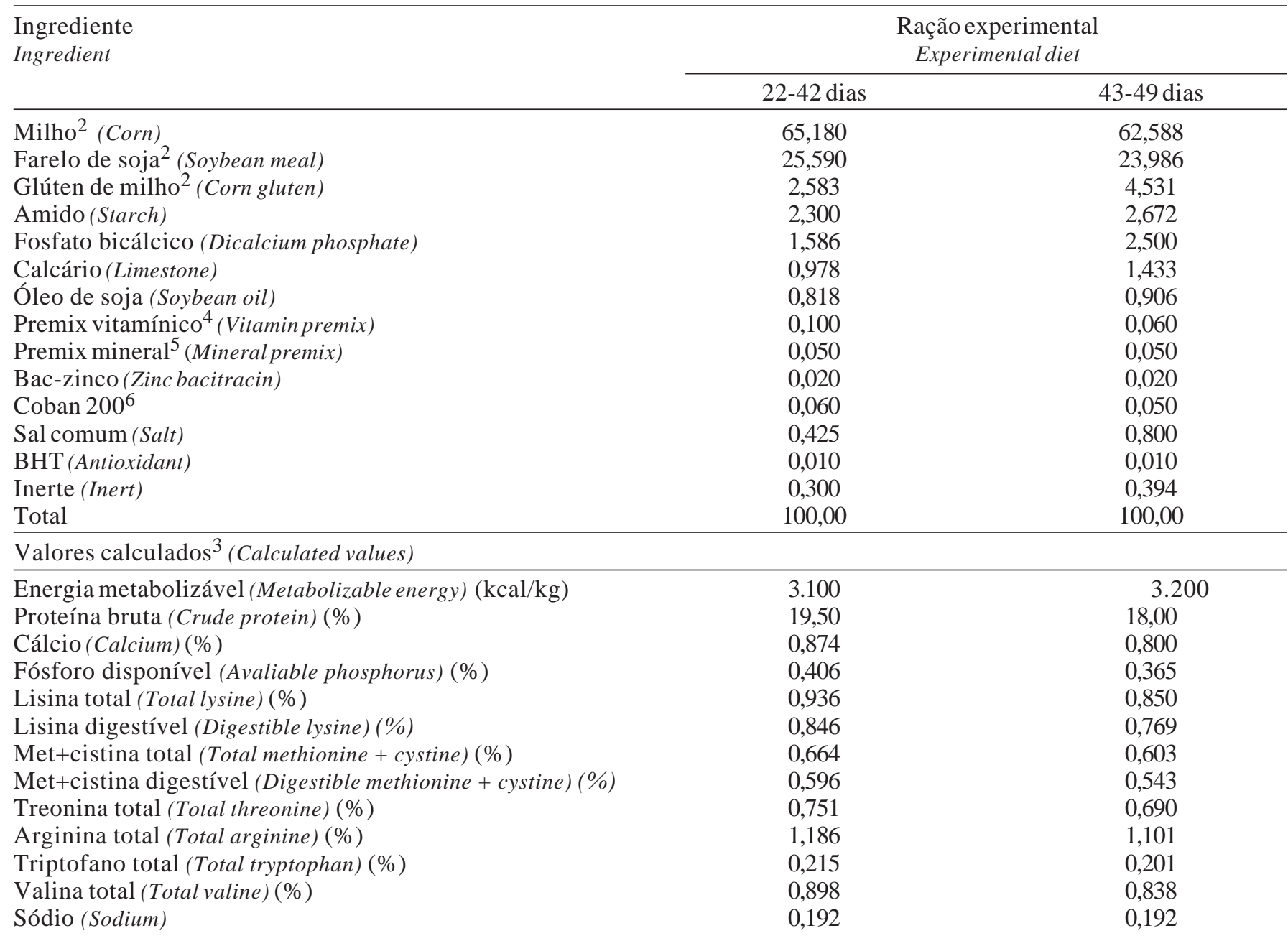

1,2, 3Valores calculados e tabelados (Rostagno et al., 2000).

1,2, 3 Tabled and calculated values (Rostagno et al., 2000).

4 Premix vitamínico por $\mathrm{kg}$ de ração (vitamin mix for ration $\mathrm{kg}$ ): Vit. A - 15.000.000 UI, Vit. D $-1.500 .000 \mathrm{UI}$, Vit. E - 15.000 UI, Vit. $B_{1}-2,0 \mathrm{~g}$, Vit. $B_{2}-4,0 \mathrm{~g}$, Vit B6 - 3,0 g, Vit.B 12 - 0,015 g, Ácido nicotínico (nicotinic acid) - $25 \mathrm{~g}$, Ácido pantotênico (pantothenic acid) - $10 \mathrm{~g}$, Vit. $\mathrm{K}_{3}-3,0 \mathrm{~g}$, Ácido fólico (folic acid) - 1,0 g, Bacitracina de zinco (zinc bacitracin) - $10 \mathrm{~g}$, Selênio (selenium) - $250 \mathrm{mg}$. Premix mineral por kg de ração (mineral mix for ration kg): Mn, 60 g; Fe, 80 g; Zn, 50 g; Cu, 10 g; Co, 2 g; l, 1 g; e veículo (vehicle) q.s.p., 500 g. Antioxidante (sinox) BHT - $10 \mathrm{~g}$, e veículo (vehicle) q.s.p. - $1.000 \mathrm{~g}$.

Monensina sódica (sodium monesin), $200 \mathrm{~g} / \mathrm{kg}$.

abate, e de peito, coxa, sobrecoxa com pele e ossos, peso do coração, fígado, moela e gordura abdominal, em relação à carcaça eviscerada.

As análises estatísticas foram realizadas utilizando-se análise de variância para as características avaliadas e o modelo de regressão polinomial para as estimativas da exigência de met + cis, por intermédio do programa computacional utilizado foi o SAS (SAS, 1996).

\section{Resultados e Discussão}

Os níveis de lisina, no período de 22 a 42 dias de idade, não influenciaram a mortalidade, que não apresentou efeito significativo entre os tratamentos. $\mathrm{Na}$ Tabela 3, constam as médias dos dados de desempenho dos frangos. Não houve efeito significativo dos diferentes níveis de lisina sobre o consumo de ração, corroborando os relatos de Valerio et al. (2003) e 
Mendes et al. (1997) e contrariando os resultados obtidos por Conhalato et al. (1999), que verificaram efeito quadrático dos níveis de lisina sobre o consumo de ração. $O$ ganho de peso dos frangos aos 42 dias de idade foi influenciado de forma quadrática $(\mathrm{P}<0,05)$, conforme a equação: $\hat{Y}=-0,2048+3,4353 X-$ $\left.1,5397 \mathrm{X}^{2} ; \mathrm{R}^{2}=0,9528\right)$, de forma semelhante ao observado por Valerio et al. (2003), que verificaram variação no peso final dos frangos aos 42 dias de idade, em razão dos níveis de lisina na dieta.

Os níveis de lisina proporcionaram redução quadrática $(\mathrm{P}<0,05)$ da conversão alimentar até o nível de 1,140\% (Figura 1). Segundo Par \& Summers (1991), além da energia, o desequilíbrio entre aminoácidos exerce influência significativa sobre a ingestão e a conversão do alimento. Valerio et al. (2003) verificaram redução na conversão alimentar de frangos de corte criados em condições de termoneutralidade, em função dos diferentes níveis de lisina das rações. Nascimento (2003b) afirma que melhores conversões podem ser justificadas pela maior ingestão de lisina da dieta, que promove maior crescimento muscular e ganho de peso, diminuindo a gordura e não alterando o consumo de ração, ocorrendo, portanto, maior otimização da conversão.
Na Tabela 4, estão apresentadas as médias relativas dos dados de avaliação de carcaça. Os parâmetros avaliados não foram influenciados pelo aumento dos níveis de lisina $(\mathrm{P}>0,05)$ mantendo-se a relação met+cis, estando, portanto, de acordo com os dados obtidos por Azcona et al. (2003), que não encontraram

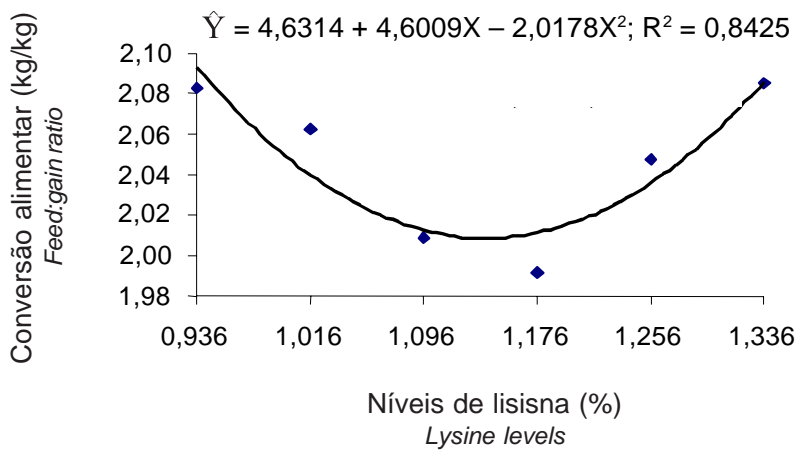

Figura 1 - Conversão alimentar de frangos de corte de 22 a 42 dias, submetidos a diferentes níveis de lisina, mantendo-se relação de $71 \%$ (met + cis:lisina).

Figure 1 - Feed:gain ratio of broilers from 22 to 42 days old on different lysine levels, maintaining $71 \%$ ratio (methionine + cystine: lysine).

Tabela 3 - Desempenho de frangos de corte de 22 a 42 dias submetidos a diferentes níveis de lisina, mantendo-se relação de $71 \%$ (met+cist:lis)

Table 3 - Performance of broilers from 22 to 42 days old on different lysine levels, maintaining 71\% ratio (met+cys:lys)

\begin{tabular}{lccc}
\hline $\begin{array}{l}\text { Nível de met + cist:lis } \\
\text { Met + cys:lys level }\end{array}$ & $\begin{array}{c}\text { Consumo de ração }(\mathrm{kg}) \\
\text { Feed intake }\end{array}$ & $\begin{array}{c}\text { Ganho de peso }(\mathrm{kg}) \\
\text { Body weight gain }\end{array}$ & $\begin{array}{c}\text { Conversão alimentar }(\mathrm{kg}) \\
\text { Feed:gain ratio }\end{array}$ \\
\hline $0,664: 0,936$ & 3,462 & 1,664 & 2,082 \\
$0,721: 1,016$ & 3,494 & 1,696 & 2,062 \\
$0,778: 1,096$ & 3,412 & 1,700 & 2,009 \\
$0,835: 1,176$ & 3,406 & 1,713 & 1,991 \\
$0,892: 1,256$ & 3,446 & 1,685 & 2,048 \\
$0,949: 1,336$ & 3,402 & 1,633 & 2,085 \\
Regressão & $\mathrm{ns}$ & $\mathrm{Q}$ & $\mathrm{Q}$ \\
CV(\%) & 2,02 & 4,11 & 3,82
\end{tabular}

ns = Efeito não-significativo $(P>0,05)$ pelo teste $F$.

$n s=$ Not significant $(P>0.05)$ effect by $F$ test.

$\mathrm{Q}^{*}=$ Efeito quadrático $(\mathrm{P}<0,01)$ do nível de Met + Cis pelo teste $\mathrm{F}$.

$Q^{*}=$ Quadratic effect $(P<0.01)$ of Met + Cys level by $F$ test. 
Tabela 4 - Valores de pesos relativos da avaliação de carcaça de frangos de corte de 22 a 42 dias, submetidos a diferentes níveis de lisina, mantendo relação de $71 \%$ (met + cist:lis)

Table 4 - Values of relative carcass weights of broilers from 22 to 42 days on different lysine levels, maintaining $71 \%$ ratio (met + cys:lys)

\begin{tabular}{lcccccccc}
\hline $\begin{array}{l}\text { Met + Cis:Lis }(\%) \\
\text { Met + Cys:Lys }\end{array}$ & $\begin{array}{c}\text { Carcaça } \\
\text { Carcass }\end{array}$ & $\begin{array}{c}\text { Peito } \\
\text { Breast }\end{array}$ & $\begin{array}{c}\text { Coxa } \\
\text { Thigh }\end{array}$ & $\begin{array}{c}\text { Sobrecoxa } \\
\text { Drumsticks }\end{array}$ & $\begin{array}{c}\text { Gordura abdominal } \\
\text { Abdominal fat }\end{array}$ & $\begin{array}{c}\text { Coração } \\
\text { Heart }\end{array}$ & $\begin{array}{c}\text { Fígado } \\
\text { Liver }\end{array}$ & $\begin{array}{c}\text { Moela } \\
\text { Gizzard }\end{array}$ \\
\hline 0,664:0,936 & 82,99 & 29,26 & 11,98 & 13,47 & 1,88 & 0,58 & 2,21 & 1,45 \\
$0,721: 1,016$ & 83,03 & 29,76 & 11,95 & 13,66 & 1,84 & 0,57 & 2,28 & 1,43 \\
0,778:1,096 & 84,07 & 29,21 & 11,76 & 13,25 & 1,70 & 0,56 & 2,24 & 1,42 \\
0,835:1,176 & 83,01 & 30,12 & 12,04 & 13,42 & 1,68 & 0,56 & 2,30 & 1,42 \\
0,892:1,256 & 84,07 & 29,90 & 11,71 & 13,16 & 1,85 & 0,57 & 2,30 & 1,42 \\
0,949:1,336 & 83,03 & 30,10 & 11,74 & 13,11 & 1,85 & 0,58 & 2,32 & 1,43 \\
Regressão & ns & ns & ns & ns & ns & ns & ns & ns \\
CV(\%) & 1,91 & 3,19 & 7,08 & 9,04 & 13,04 & 6,22 & 5,19 & 4,89 \\
\hline
\end{tabular}

ns = Efeito não-significativo $(P>0,05)$ pelo teste $F$.

$n s=$ Not significant $(P>0.05)$ effect by $F$ test.

efeito significativo sobre o rendimento de carcaça, peito e gordura abdominal e trabalharam com uma relação de 73\% (met + cis:lis). No entanto, a maioria dos trabalhos realizados com lisina, sem manter relação, demonstram alteração principalmente no rendimento de peito e na deposição de gordura abdominal (Nascimento, 2003a, Borges et al., 2002; Barboza, 1998) em relação à lisina dietética.

Níveis de met + cis da dieta no desempenho e na avaliação de carcaça de frangos de corte no período de 43 a 49 dias de idade.

A mortalidade, no período de 43 a 49 dias de idade, foi de $0,97 \%$ sem efeito significativo dos tratamentos $(\mathrm{P}>0,05)$. Na Tabela 5, estão apresentadas os valores médios do desempenho dos frangos.

Houve efeito quadrático $(\mathrm{P}<0,01)$ dos níveis de lisina na dieta sobre a conversão alimentar e o ganho de peso (Figura 2), conforme as seguintes equações: $\hat{Y}=15,363-25,427 X+12,182 X^{2} ; R^{2}=0,7908 e$ $\hat{Y}=4,0789+9,2007 X-4,4922 X^{2} ; R^{2}=0,6771$, respectivamente. Apesar de o consumo de ração não ter apresentado efeito significativo, o ganho de peso teve resposta favorável, correspondendo à melhor eficiência de utilização da dieta quando estabelecidas as relações próximas às exigências. Os valores de exigências encontrados estão bastante próximos à exigência de lisina verificada em outro experimento quando não foi mantida a relação com aminoácidos sulfurosos (Amarante Jr., dados não publicados). Porém, Valerio et al. (2003), trabalhando com a fase de 22 a 42 dias, verificaram que a exigência quando se mantêm a relação é maior e observaram que a manutenção da relação favorece a conversão alimentar.
Neste contexto, Lemme (2003) afirma que o desempenho das aves varia com a idade e com a relação que é estabelecida entre met + cis/lis, e que resultados diferenciados são encontrados com relações de 72 a $82 \%$, de acordo com a fase de criação.

Na Tabela 6, estão apresentadas as médias relativas dos dados de avaliação de carcaça. Observouse que os níveis de lisina da dieta influenciaram de forma quadrática o rendimento de carcaça dos frangos aos 49 dias, estimando-se exigência de 1,035\% de lisina total, conforme a equação $\hat{Y}=-24,184+$ $209,43 X-101,12 X^{2} ; R^{2}=0,6774$. Estes resultados

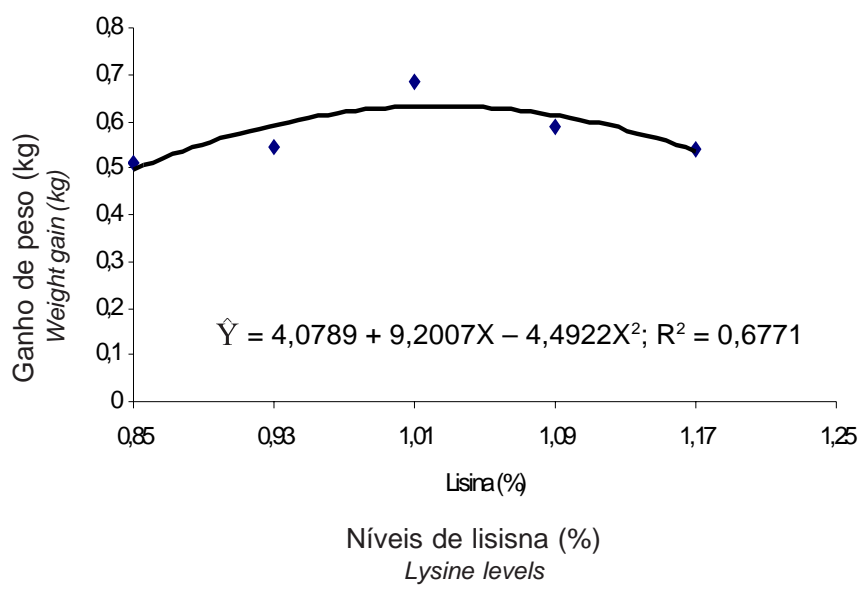

Figura 2 - Ganho de peso de frangos de corte submetidos a diferentes níveis lisina, mantendo relação de 71\% (met+cis:lis) de 43 a 49 dias.

Figure 2 - Body weight gain of broilers from 43 to 49 days old on different lysine levels, maintaining $71 \%$ ratio (methionine + cystine:lysine) 
Tabela 5 - Desempenho de frangos de corte submetidos a diferentes níveis de lisina, mantendo relação de $71 \%$ (metionina+cistina: lisina) de 43 a 49 dias

Table 5 - Performance of broilers from 43 to 49 days old on different lysine levels, maintaining $71 \%$ ratio (met+cys:lys)

\begin{tabular}{lccc}
\hline $\begin{array}{l}\text { Nível de metionina + cistina:lisina } \\
\text { Methionine + cystine level:lysine }\end{array}$ & $\begin{array}{c}\text { Consumo de ração }(\mathrm{kg}) \\
\text { Feed intake }\end{array}$ & $\begin{array}{c}\text { Ganho de peso }(\mathrm{kg}) \\
\text { Body weight gain }\end{array}$ & $\begin{array}{c}\text { Conversão alimentar }(\mathrm{kg}) \\
\text { Feed:gain ratio }\end{array}$ \\
\hline 0,603:0,850 & 1,286 & 0,511 & 2,541 \\
$0,660: 0,930$ & 1,276 & 0,544 & 2,352 \\
$0,717: 1,010$ & 1,311 & 0,685 & 1,915 \\
$0,774: 1,090$ & 1,275 & 0,589 & 2,216 \\
$0,831: 1,171$ & 1,258 & 0,539 & 2,340 \\
$0,888: 1,250$ & 1,317 & 0,513 & 2,576 \\
Regressão & $\mathrm{ns}$ & $\mathrm{Q} *$ & $\mathrm{Q}^{*}$ \\
CV(\%) & 7,07 & 12,26 & 8,56
\end{tabular}

ns = Efeito não-significativo $(P>0,05)$ pelo teste $F$.

$n s=$ Not significant $(P>0.05)$ effect by $F$ test.

$\mathrm{Q}^{*}=$ Efeito quadrático $(\mathrm{P}<0,01)$ do nível de Met + Cis pelo teste $\mathrm{F}$

$Q^{*}=$ Quadratic effect $(P<0.01)$ of Met + Cys level by $F$ test.

Tabela 6 - Valores de pesos relativos da avaliação de carcaça de frangos de corte de 43 a 49 dias submetidos a diferentes níveis de lisina, mantendo-se relação de $71 \%$ (met +cis:lisina)

Table 6 - Values of relative carcass weights of broilers from 43 to 49 days old on different lysine levels, maintaining $71 \%$ ratio (met+cys:lys)

\begin{tabular}{lcccccccc}
\hline $\begin{array}{l}\text { Met + Cis:Lis } \\
\text { Met + Cys:Lis (\%) }\end{array}$ & $\begin{array}{c}\text { Carcaça } \\
\text { Carcass }\end{array}$ & $\begin{array}{c}\text { Peito } \\
\text { Breast }\end{array}$ & $\begin{array}{c}\text { Coxa } \\
\text { Thigh }\end{array}$ & $\begin{array}{c}\text { Sobrecoxa } \\
\text { Drumsticks }\end{array}$ & $\begin{array}{c}\text { Gordura abdominal } \\
\text { Abdominal fat }\end{array}$ & $\begin{array}{c}\text { Coração } \\
\text { Heart }\end{array}$ & $\begin{array}{c}\text { Fígado } \\
\text { Liver }\end{array}$ & $\begin{array}{l}\text { Moela } \\
\text { Gizzard }\end{array}$ \\
\hline 0,603:0,850 & 88,79 & 30,45 & 12,44 & 14,28 & 1,95 & 0,48 & 2,05 & 1,54 \\
$0,660: 0,930$ & 82,24 & 30,67 & 12,15 & 14,70 & 2,25 & 0,51 & 2,13 & 1,53 \\
$0,717: 1,010$ & 86,39 & 31,00 & 12,24 & 14,94 & 1,97 & 0,52 & 2,03 & 1,55 \\
$0,774: 1,090$ & 82,51 & 30,88 & 11,78 & 14,56 & 2,14 & 0,54 & 2,05 & 1,56 \\
$0,831: 1,170$ & 82,20 & 30,44 & 12,07 & 14,33 & 1,96 & 0,50 & 1,92 & 1,57 \\
0,888:1,250 & 79,96 & 30,11 & 12,04 & 14,76 & 1,82 & 0,51 & 2,04 & 1,56 \\
Regressão & $\mathrm{Q}^{*}$ & $\mathrm{Q}^{* *}$ & $\mathrm{~ns}$ & $\mathrm{~ns}$ & $\mathrm{~ns}$ & $\mathrm{Q}$ & $\mathrm{n}$ & $\mathrm{ns}$ \\
CV(\%) & 4,31 & 1,59 & 4,37 & 4,31 & 18,37 & 6,81 & 7,54 & 3,05 \\
\hline
\end{tabular}

ns = Efeito não-significativo $(P>0,05)$ pelo teste $F$.

$n s=$ Not significant $(P>0.05)$ effect by $F$ test.

$\mathrm{Q}^{*}$ e $\mathrm{Q}^{\star *}=$ Efeito quadrático do nível de met+cis:lis pelo test $\mathrm{F}(\mathrm{P}<0,05$ e $\mathrm{P}<0,01)$, respectivamente.

$Q^{*}$ and $Q^{* *}=$ Quadratic effect of met+cys:lys level by $F$ test $(P<0.05$ and $P<0.01)$, respectively.

estão de acordo com Bilgili et al. (1992), ao afirmarem que o aumento do nível de lisina na fase final de criação aumenta o ganho de peso da ave e o rendimento de carcaça, principalmente carne de peito. Entretanto, Costa et al. (2001) e Barboza \& Rostagno (1998) não verificaram efeito dos níveis de lisina sobre o rendimento de carcaça, cortes nobres e gordura abdominal.

Os níveis de lisina influenciaram o rendimento de peito de forma quadrática $(\mathrm{P}<0,01)$ com exigência estimada em $1,023 \%$ de lisina total, conforme a seguinte equação $\hat{\mathrm{Y}}=13,885+33,281 \mathrm{X}-16,26 \mathrm{X}^{2}$; $\left.\mathrm{R}^{2}=0,93\right)$. Azcona et al. (2003) encontraram exigência de 1,07\% de lisina digestível, mantendo-se relação em 73\% de met + cis:lis. Moran Jr. \& Bilgili (1990), estudando efeitos da suplementação de lisina sobre as características e a composição da carcaça de frangos de corte na fase final de criação, determinaram exigência média de 0,85 a 1,05\% de lisina total para conversão alimentar, rendimento de peito e gordura abdominal. Rezende et al. (1980) verificaram exigência média de 0,85 a 0,95\% em função dos níveis de lisina:aminoácidos sulfurosos.

Os níveis de lisina influenciaram significativamente, de forma quadrática $(\mathrm{P}<0,05)$, o peso relativo do coração dos frangos, que aumentou até o nível de $1,087 \%$ de lisina total, conforme a seguintes equação $\left.\hat{\mathrm{Y}}=-0,4054+1,7197 \mathrm{X}-0,7906 \mathrm{X}^{2} ; \mathrm{R}^{2}=0,754\right)$. 
Valério et al. (2003) observaram efeito quadrático sobre o peso do coração de frangos, que reduziu até o nível de 1,08\% de lisina digestível em dietas convencionais. O resultado observado neste estudo evidenciou que o crescimento do coração, órgão muscular, pode ter sido influenciado pelo nível de lisina a partir da síntese de proteína muscular.

\section{Conclusões}

Os dados de desempenho e de avaliação de carcaça, nas condições em que foram realizados os dois experimentos, demonstram que é possível a utilização de níveis nutricionais de 1,140\% de lisina total (1,03\% de lisina digestível) para frangos de corte machos da linhagem Ross, no período de 22 a 42 dias de idade, e 1,024\% (0,926\% de lisina digestível), para a fase de 43 a 49 dias, mantendo-se relação em 71\% (met+cis:lis).

\section{Literatura Citada}

AZCONA, J.; SCHANG, M.; CORTAMIRA, O. Nível de lisina nas rações de frangos de corte: Experimento 1 - 22 a 42 dias de idade, Experimento 2 - 36 a 49 dias de idade. Relatório de Pesquisa - 35. Ajinomoto Animal Nutrition Ajinomoto Biolatina Ind. e Com.Ltda. p.1 - 7, 2003. Disponível em : www.lisina.com.br, acessado em 30 de maio de 2004.

BARBOZA, W.A. Exigências nutricionais de lisina para duas marcas comerciais de frangos de corte. Viçosa, MG: Universidade Federal de Viçosa, 1998. 116p. Tese (Doutorado em Zootecnia) - Universidade Federal de Viçosa.

BARBOZA, W.A.; ROSTAGNO, H.S. Exigências nutricionais de lisina para frangos de corte no período de 15 a 40 dias de idade. In: REUNIÃO ANUAL DA SOCIEDADE BRASILEIRA DE ZOOTECNIA, 35., 1998, Botucatu. Anais... Botucatu: Sociedade Brasileira de Zootecnia, 1998. p.502504.

BILGILI, S.F.; MORAN JR., E.T.; ACAR, N. Strain cross response of heavy male broilers to dietary lysine in the finisher feed: live performance and further-processing yields. Poultry Science, v.71, p.850-858, 1992.

BORGES, A.F.; OLIVEIRA, R.F.M.; DONZELE, J.L. et al. Exigência de lisina para frangos de corte machos no período de 22 a 42 dias de idade, mantidos em ambiente quente $\left(26^{\circ} \mathrm{C}\right)$. Revista Brasileira de Zootecnia, v.31, n.5, p.1993-2001, 2002.

CONHALATO, G.S.; DONZELE, J.L.; ALBINO, L.F.T. et al. Níveis de lisina digestível para frangos de corte machos na fase de 21 a 42 dias de idade. Revista Brasileira de Zootecnia, v.28, n.1, p.91-97, 1999.
COSTA, F.G.P.; ROSTAGNO, H.S.; ALBINO, L.F.T. et al. Níveis de lisina digestível para frangos de corte machos na fase de 21 a 42 dias de idade. Revista Brasileira de Zootecnia, v.30, n.5, p.1490-1497, 2001.

LEMME, A. El “Concepto de Proteína ideal” en la nutrición de pollos. 1. Aspectos metodológicos - Oportunidades y limitaciones. AminoNews TM, v.4, n.1, p.7-18, 2003.

MENDES, A.A.; WATKINS, S.E.; ENGLAND, J.A. et al. Influence of dietary lysine levels and arginine: lysine ratios on performance of broilers exposed to heat or cold stress during the period of three to six weeks of age. Poultry Science, v.76, p.472-481, 1997.

MORAN JR., E.T.; BILGILI, S.F. Processing losses, carcass quality and meat yields for broiler chicken, receiving diets marginally deficient to adequate in lysine prior to marketing. Poultry Science, v.69, p.702-710, 1990.

NASCIMENTO, A. Deposição de proteína na carcaça de frangos de corte. Revista Ave World, n.2, p.2-3, 2004.

NASCIMENTO, A. Lisina - Principal aminoácido para deposição protéica. Revista Ave World, ano 1, n.1, p.56-61, $2003 a$.

NASCIMENTO, A. Lisina otimiza a conversão alimentar. Revista Ave World, ano 1, n.5, p.48-50, 2003b.

PARR, J.F.; SUMMERS, J.D. The effects of minimizing amino acid excess in broiler diets. Poultry Science, v.70, p.1540-549, 1991.

REZENDE, J.A.A.; ROSTAGNO, H.S.; SILVA, M.A. et al. Níveis de proteína, aminoácidos sulfurosos e lisina em rações de frangos submetidos a regime de alta temperatura. Fase final. Revista da Sociedade Brasileira de Zootecnia, v.9, n.1, p.125-14l, 1980b.

ROSTAGNO, H.S.; SILVA, D.J.; COSTA, P.M.A. et al. Composição de alimentos e exigências nutricionais de aves $e$ suínos (Tabelas Brasileiras). Viçosa, MG: Universidade Federal de Viçosa, 2000. 141p.

SCOTT, M.L.; NESHEIM, M.C.; YOUNG, R.J. Nutrition of the chicken. 3.ed. New York: Ed. Ithaca, 1982. 562p.

SKLAN, D.; NOY, Y. Catabolism and deposition of amino acids in growing chicks: effect of dietary supply. Poultry Science, v.83, p.952-961, 2004.

STATISTICAL ANALYSES SYSTEM - SAS. User's guide: statistics. 12.ed. New York: SCOTT, M.L. \& Associates. 1996. 511p.

VALÉRIO, S.R.; OLIVEIRA, R.F.M.; DONZELE, J.L. et al. Níveis de lisina digestível em rações, mantendo ou não a relação aminoacídica, para frangos de corte de 22 a 42 dias de idade, sob condições de estresse por calor. Revista Brasileira de Zootecnia, v.32, n.2, p.372-382, 2003. 